MCTP-10-45

\title{
Supersymmetric massive truncations of IIB supergravity on Sasaki-Einstein manifolds
}

\author{
James T. Liu * Phillip Szepietowski $\ddagger$ and Zhichen Zhad \\ Michigan Center for Theoretical Physics, Randall Laboratory of Physics, \\ The University of Michigan, Ann Arbor, MI 48109-1040, USA
}

\begin{abstract}
Motivated by recent interest in applications of the AdS/CFT correspondence to condensed matter applications involving fermions, we present the supersymmetric completion of the recent massive truncations of IIB supergravity on Sasaki-Einstein manifolds. In particular, we reduce the fermionic sector of IIB supergravity to obtain five dimensional $\mathcal{N}=2$ supergravity coupled to one hypermultiplet and one massive vector multiplet. The supersymmetry transformations and equations of motion are presented and analyzed. Finally, a particularly interesting truncation to $\mathcal{N}=2$ supergravity coupled to a single hypermultiplet is presented which is the supersymmetric completion of the recently constructed bosonic theory dual to a $3+1$ dimensional system exhibiting a superconducting phase transition.
\end{abstract}

*Electronic address: jimliu@umich.edu

$\dagger$ Electronic address: pszepiet@umich.edu

${ }^{\ddagger}$ Electronic address: zhichen@umich.edu 


\section{INTRODUCTION}

While the study of consistent Kaluza-Klein truncations has a rich history, until now much of it has focused on reductions that retain only the massless sector of the lower dimensional theory. This may be partially attributed to the standard lore that it would be inconsistent to retain a finite number of states in the Kaluza-Klein tower without pulling in the rest. However, a simple method of evading this difficulty is to retain only singlets of a transitively acting subgroup of the internal symmetry group. A simple example of this prescription is the inclusion of breathing and possibly squashing modes, and for sphere compactifications the reductions were explicitly constructed in [1].

The basic breathing mode compactification of [1] was obtained by truncating to singlets on spheres. Hence the resulting theories were necessarily non-supersymmetric. Nevertheless, as long as the underlying theories (such as IIB or $D=11$ supergravity) are supersymmetric, it would still be fair to investigate the supersymmetry of breathing mode backgrounds. This was carried out in [2], where it was demonstrated that the original fermionic variations of IIB and $D=11$ supergravity reduce to their effective $\mathcal{N}=2$ counterparts in five dimensions (for IIB supergravity on a squashed $S^{5}$ ) and four dimensions (for $D=11$ supergravity on a squashed $S^{7}$ ), respectively. In fact, in both cases it was possible to read off an effective $\mathcal{N}=2$ superpotential from the lower dimensional gravitino variations. Moreover it was conjectured in [2] that supersymmetric consistent truncations may be obtained by retaining singlets not under the full isometry groups $\mathrm{SO}(8)$ and $\mathrm{SO}(6)$, but rather subgroups $\mathrm{SU}(4)$ and $\mathrm{SU}(3)$, for the squashed $S^{7}$ and $S^{5}$, respectively.

The consistent truncation conjecture of [2] was subsequently verified for $D=11$ supergravity on a squashed Sasaki-Einstein manifold by explicit construction in [3]. The reduction was performed by writing $S E_{7}$ as U(1) bundled over a Kahler-Einstein base and then expanding the four-form field strength in a basis of invariant tensors corresponding to the $\mathrm{SU}(3)$ structure on the base. The closure of the $\mathrm{SU}(3)$ structure equations then ensures the consistency of the truncation. More recently, similar constructions have been obtained for IIB supergravity on $S E_{5}[4-7]$ and $T^{1,1}[8,9]$. A curious feature of these reductions is that, while the standard reduction of IIB on $S E_{5}$ yields ordinary $\mathcal{N}=2$ gauged supergravity in five dimensions, the massive truncation retains a massive gravitino multiplet, and hence ought to be viewed as a spontaneously broken $\mathcal{N}=4$ theory. 
While the consistent truncation procedure in these cases is guaranteed to preserve supersymmetry, until now much of the focus has been on the bosonic sectors. Nevertheless it would be useful to have an explicit realization of the fermion reduction as well. This is especially interesting in light of holographic models of superconductivity in $2+1$ [10, 11] and $3+1$ [12] dimensions, where electronic properties often involve fermion correlators and not just the bosons. Along these lines, the fermion sector of the reduction of $D=11$ supergravity on squashed $S E_{7}$ was recently constructed in [13]. The procedure is similar to that used in the bosonic reduction. In particular, the eleven-dimensional fermions may be expanded in terms of invariant tensors multiplying Killing spinors. This naturally retains the lowest modes in spinor harmonics in each of the Kaluza-Klein towers, and ensures the overall consistency of the reduction.

In this paper, we focus on the $\mathcal{N}=2$ truncation of IIB supergravity reduced on squashed $S E_{5}$, and demonstrate the consistent reduction of the fermion sector, at least to quadratic order in the fermions. As demonstrated in [4 7], the full bosonic sector of this reduction corresponds to an $\mathcal{N}=4$ theory. However, by truncating out the $\mathcal{N}=2$ massive gravitino multiplet, we may bring this down to $\mathcal{N}=2$. While our main motivation for doing so is to avoid unnecessarily cumbersome expressions related to the massive gravitino sector, we do not see any obstacles to achieving the full reduction if desired. Furthermore, this allows us to highlight some of the features of the reduction from an $\mathcal{N}=2$ perspective.

Since the reduction of the fermionic sector uses the bosonic reduction as a starting point, we begin with a brief review of the bosons in Section II. We then turn to the reduction of the IIB fermions in Section [II and present the effective five-dimensional theory in Section IV] Moreover, as shown in Section $\square$, the resulting $\mathcal{N}=2$ theory admits a truncation to gauged supergravity coupled to a single hypermultiplet, corresponding to the model of [12] for a holographic superconductor in $3+1$ dimensions.

While this work was being completed we became aware of [14], which has substantial overlap with our results. In fact, [14] has worked out the reduction of the fermions corresponding to the full $\mathcal{N}=4$ theory, thus demonstrating consistency of the complete massive truncation, and not just the $\mathcal{N}=2$ sector that we focus on here. 


\section{THE BOSONIC REDUCTION OF IIB SUPERGRAVITY ON $S E_{5}$}

The reduction of the bosonic sector of IIB supergravity on a squashed Sasaki-Einstein manifold was carried out in [4-7]. From an $\mathcal{N}=2$ point of view, the resulting theory has on-shell fields corresponding to that of five-dimensional gauged supergravity coupled to a massive hypermultiplet, massive gravitino multiplet and massive vector multiplet [2, 5].

Before turning to the fermions, we review the reduction of the bosonic sector, following the notations and conventions of [5]. Although IIB supergravity does not admit a covariant action, we may take a bosonic Lagrangian of the form

$$
\mathcal{L}_{\mathrm{IIB}}=R * 1-\frac{1}{2 \tau_{2}^{2}} d \tau \wedge * d \bar{\tau}-\frac{1}{2} \mathcal{M}_{i j} F_{3}^{i} \wedge * F_{3}^{j}-\frac{1}{4} \widetilde{F}_{5} \wedge * \widetilde{F}_{5}-\frac{1}{4} \epsilon_{i j} C_{4} \wedge F_{3}^{i} \wedge F_{3}^{j},
$$

where self-duality $\widetilde{F}_{5}=* \widetilde{F}_{5}$ is to be imposed by hand after deriving the equations of motion. Here we have chosen to write the Lagrangian in an $\mathrm{SL}(2, \mathbb{R})$ invariant form using

$$
\tau=C_{0}+i e^{-\phi}, \quad \mathcal{V}=\frac{1}{\sqrt{\tau_{2}}}\left(\begin{array}{cc}
-\tau_{1} & 1 \\
\tau_{2} & 0
\end{array}\right), \quad \mathcal{M}=\mathcal{V}^{T} \mathcal{V}=\frac{1}{\tau_{2}}\left(\begin{array}{cc}
|\tau|^{2} & -\tau_{1} \\
-\tau_{1} & 1
\end{array}\right)
$$

For convenience when coupling to fermions, we also introduce the complexified vielbein $v_{i}=\mathcal{V}^{1}{ }_{i}-i \mathcal{V}^{2}{ }_{i}$, so that

$$
v_{i} F_{3}^{i}=\tau_{2}^{-1 / 2}\left(F_{3}^{2}-\tau F_{3}^{1}\right)=\tau_{2}^{-1 / 2} G_{3},
$$

where $G_{3}=F_{3}^{2}-\tau F_{3}^{1}$.

The reduction ansatz follows by taking a metric of the squashed Sasaki-Einstein form

$$
d s_{10}^{2}=e^{2 A} d s_{5}^{2}+e^{2 B} d s^{2}(B)+e^{2 C}\left(\eta+A_{1}\right)^{2},
$$

where $d \eta=2 J$ and where we set $3 A+4 B+C=0$ to remain in the Einstein frame. The key to the reduction is to expand the remaining bosonic fields in terms of the invariant forms $J$ and $\Omega$ based on the $\mathrm{SU}(2)$ structure of the base $B$ and satisfying

$$
J \wedge \Omega=0, \quad \Omega \wedge \bar{\Omega}=2 J \wedge J=4 *_{4} 1, \quad *_{4} J=J, \quad *_{4} \Omega=\Omega,
$$

as well as

$$
d J=0, \quad d \Omega=3 i(d \psi+\mathcal{A}) \wedge \Omega
$$

The bosonic reduction follows by expanding the three-form and five-form field strengths in a basis of invariant tensors on $B$. Since we will truncate out the massive gravitino multiplet, 
we set the corresponding bosonic fields to zero. (The complete reduction is given in [5].) In this case, the three-form gives rise to two complex scalars $b^{i}$, and is given by

$$
F_{3}^{i}=f_{1}^{i} \wedge \Omega+\bar{f}_{1}^{i} \wedge \bar{\Omega}+f_{0}^{i} \wedge \Omega \wedge\left(\eta+A_{1}\right)+\bar{f}_{0}^{i} \wedge \bar{\Omega} \wedge\left(\eta+A_{1}\right)
$$

where

$$
f_{1}^{i}=D b^{i}, \quad f_{0}^{i}=3 i b^{i}
$$

with $D$ the $\mathrm{U}(1)$ gauge covariant derivative

$$
D b^{i}=d b^{i}-3 i A_{1} b^{i}
$$

Furthermore, introducing

$$
b^{i}=\left(\begin{array}{l}
1 \\
\tau
\end{array}\right) b^{m^{2}=-3}+\left(\begin{array}{l}
1 \\
\bar{\tau}
\end{array}\right) b^{m^{2}=21},
$$

it is easy to see that

$$
v_{i} f_{0}^{i}=6 \sqrt{\tau_{2}} b^{m^{2}=21}, \quad \bar{v}_{i} f_{0}^{i}=-6 \sqrt{\tau_{2}} b^{m^{2}=-3},
$$

while

$$
\begin{aligned}
& v_{i} f_{1}^{i}=-2 i \sqrt{\tau_{2}}\left[D b^{m^{2}=21}+\frac{i}{2 \tau_{2}}\left(b^{m^{2}=-3} d \tau+b^{m^{2}=21} d \bar{\tau}\right)\right] \\
& \bar{v}_{i} f_{1}^{i}=2 i \sqrt{\tau_{2}}\left[D b^{m^{2}=-3}-\frac{i}{2 \tau_{2}}\left(b^{m^{2}=-3} d \tau+b^{m^{2}=21} d \bar{\tau}\right)\right]
\end{aligned}
$$

These expressions will show up extensively in the fermion reduction below.

For the self-dual five-form, we have

$$
\widetilde{F}_{5}=(1+*)\left[\left(4+\phi_{0}\right) *_{4} 1 \wedge\left(\eta+A_{1}\right)+\mathbb{A}_{1} \wedge *_{4} 1+p_{2} \wedge J \wedge\left(\eta+A_{1}\right)\right],
$$

where $*_{4} 1$ denotes the volume form on the Kahler-Einstein base $B$. The fields $\phi_{0}$ and $p_{2}$ are constrained by

$$
\begin{aligned}
\phi_{0} & =-\frac{2 i}{3} \epsilon_{i j}\left(f_{0}^{i} \bar{f}_{0}^{j}-\bar{f}_{0}^{i} f_{0}^{j}\right), \\
p_{2} & =-d\left[A_{1}+\frac{1}{4} \mathbb{A}_{1}+\frac{i}{6} \epsilon_{i j}\left(f_{0}^{i} \bar{f}_{1}^{j}-\bar{f}_{0}^{i} f_{1}^{j}\right)\right] .
\end{aligned}
$$

Hence the only additional field arising from the five-form is the vector $\mathbb{A}_{1}$. 
Finally, we note that the bosonic field content of this massive truncation is that of gauged supergravity coupled to a hypermultiplet with fields $\left(\tau, b^{m^{2}=-3}\right)$ and a massive vector multiplet with fields $\left(B, C, b^{m^{2}=21}, \mathbb{A}_{1}\right)$. This massive multiplet is actually a vector combined with a hypermultiplet. However, since we are working on shell, one of the scalars has been absorbed into the massive vector. If desired, this scalar may be restored by an appropriate Stueckelberg shift of $\mathbb{A}_{1}$.

\section{REDUCTION OF THE IIB FERMIONS}

We are now prepared to examine the fermionic sector of IIB supergravity [15]. For simplicity in working out the reduction, we follow a Dirac convention throughout. In this case, the fermions consist of a spin- $\frac{3}{2}$ gravitino $\Psi_{M}$ and a spin- $\frac{1}{2}$ dilatino $\lambda$, with opposite chiralities

$$
\Gamma_{11} \Psi_{M}=\Psi_{M}, \quad \Gamma_{11} \lambda=-\lambda .
$$

Our Dirac conventions are detailed in Appendix A. In particular, as opposed to [15], we are using a mostly plus metric signature.

In the following we always work to lowest order in the fermions. In this case, the IIB supersymmetry variations on the fermions are given by [15]

$$
\begin{gathered}
\delta \lambda=\frac{i}{2 \tau_{2}} \Gamma^{A} \partial_{A} \tau \epsilon^{c}-\frac{i}{24} \Gamma^{A B C} v_{i} F_{A B C}^{i} \epsilon \\
\delta \Psi_{M}=\mathcal{D}_{M} \epsilon \equiv\left(\nabla_{M}+\frac{i}{4 \tau_{2}} \partial_{M} \tau_{1}+\frac{i}{16 \cdot 5 !} \Gamma^{A B C D E} \widetilde{F}_{A B C D E} \Gamma_{M}\right) \epsilon \\
+\frac{i}{96}\left(\Gamma_{M}{ }^{A B C}-9 \delta_{M}^{A} \Gamma^{B C}\right) v_{i} F_{A B C}^{i} \epsilon^{c}
\end{gathered}
$$

The supersymmetry parameter $\epsilon$ is chiral with $\Gamma_{11} \epsilon=\epsilon$, and the complexified $S L(2, \mathbb{R})$ vielbein, $v_{i}$, was defined above in (3). In addition the fermion equations of motion are [15]

$$
\begin{aligned}
& 0=\Gamma^{M} \mathcal{D}_{M} \lambda-\frac{i}{8 \cdot 5 !} \Gamma^{M N P Q R} F_{M N P Q R} \lambda, \\
& 0=\Gamma^{M N P} \mathcal{D}_{N} \Psi_{P}+\frac{i}{48} \Gamma^{N P Q} \Gamma^{M} v_{i}^{*} F_{N P Q}^{i *} \lambda-\frac{i}{4 \tau_{2}} \Gamma^{N} \Gamma^{M} \partial_{N} \tau \lambda^{c},
\end{aligned}
$$

where the supercovariant derivative acting on the gravitino is defined in the gravitino variation (16). On the other hand, the supercovariant derivative acting on the dilatino takes the form

$$
\mathcal{D}_{M} \lambda=\left(\nabla_{M}+\frac{3 i}{4 \tau_{2}} \partial_{M} \tau_{1}\right) \lambda-\frac{i}{2 \tau_{2}} \Gamma^{N} \partial_{N} \tau \Psi_{M}^{c}+\frac{i}{24} \Gamma^{N P Q} v_{i} F_{N P Q}^{i} \Psi_{M}
$$


and is defined so that $\nabla_{M} \epsilon$ terms drop out of the variation $\mathcal{D}_{M} \delta \lambda$, as appropriate to supercovariantization.

\section{A. Killing spinors on $S E_{5}$}

The starting point of the fermion reduction is the construction of Killing spinors on $S E_{5}$. Starting with the undeformed Sasaki-Einstein metric

$$
d s^{2}\left(S E_{5}\right)=d s^{2}(B)+(d \psi+\mathcal{A})^{2}
$$

the Killing spinor equations then follow from the internal components of the gravitino variation in (16) with a constant five-form flux

$$
\tilde{F}_{5}=4 *_{5} 1+4 *_{4} 1 \wedge(d \psi+\mathcal{A})
$$

and take the form

$$
\begin{aligned}
& 0=\delta \Psi_{a}=\hat{\mathcal{D}}_{a} \eta \equiv\left[\hat{\nabla}_{a}-\mathcal{A}_{a} \partial_{\psi}+\frac{1}{2} J_{a b} \tau^{b} \tau^{9}+\frac{i}{2} \tau_{a}\right] \eta \\
& 0=\delta \Psi_{9}=\left[\partial_{\psi}-\frac{1}{4} J_{a b} \tau^{a b}+\frac{i}{2} \tau_{9}\right] \eta
\end{aligned}
$$

We proceed by assigning a $\mathrm{U}(1)$ charge $q$ to the Killing spinor $\eta$, so that $\partial_{\psi} \eta=i q \eta$. Furthermore, since $\left(J_{a b} \tau^{a b}\right)^{2}=-8\left(1-\tau^{9}\right)$, we see that $J_{a b} \tau^{a b}$ has eigenvalues $(4 i,-4 i, 0,0)$ with corresponding $\tau^{9}$ eigenvalues $(-1,-1,1,1)$. The variation $\delta \Psi_{9}$ then vanishes for the charges $q=\left(\frac{3}{2},-\frac{1}{2},-\frac{1}{2},-\frac{1}{2}\right)$. The $\mathcal{N}=2$ Killing spinor is thus obtained by taking $q=\frac{3}{2}$ and $J_{a b} \tau^{a b} \eta=4 i \eta$.

Having exhausted the content of the $\delta \Psi_{9}$ equation, we now turn to integrability of $\delta \Psi_{a}$, which gives the requirement

$$
0=\tau^{b}\left[\hat{\mathcal{D}}_{a}, \hat{\mathcal{D}}_{b}\right] \eta=\tau^{b}\left[\delta_{a b}\left(\tau^{9}-1\right)-i J_{a b}\left(\tau^{9}+2 q\right)\right] \eta
$$

For $q=\frac{3}{2}$ and $\tau^{9} \eta=-\eta$, this gives the condition $J_{a b} \tau^{b} \eta=i \tau_{a} \eta$, which is easily seen to be consistent with the above requirement that $J_{a b} \tau^{a b} \eta=4 i \eta$. After defining $\eta=e^{3 i \psi / 2} \tilde{\eta}$, we are finally left with the condition

$$
\left[\hat{\nabla}_{a}-\frac{3 i}{2} \mathcal{A}_{a}\right] \tilde{\eta}=0
$$

which is solved by taking $\tilde{\eta}$ to be a gauge covariantly constant spinor on the Kahler-Einstein base [16]. 
To summarize the above, the system (21) may be solved to yield a single complex Killing spinor $\eta$ satisfying

$$
\partial_{\psi} \eta=\frac{3 i}{2} \eta, \quad \tau^{9} \eta=-\eta, \quad \tau^{b} J_{a b} \eta=i \tau_{a} \eta, \quad \tau^{b} \Omega_{a b} \eta=0 .
$$

The final condition may be obtained by multiplying the penultimate one by $\Omega_{c a}$ on both sides and making use of the identity $\Omega_{c a} J_{a b}=-i \Omega_{c b}$, which follows from the relations [6]

$$
\Omega_{a c} \Omega^{b c}=0, \quad \Omega_{a c} \bar{\Omega}^{b c}=2 \delta_{a}^{b}-2 i J_{a}^{b} .
$$

The Killing spinor $\eta$ and its conjugate $\eta^{c}$ provide a natural basis of invariant spinors in which to expand the fermions. Furthermore, as discussed in [13], these represent singlets of the $S U(2)$ structure group, thus ensuring consistency of the reduction. Note that $\eta$ and $\eta^{c}$ are related by

$$
\tau^{b} \bar{\Omega}_{a b} \eta=2 \tau_{a} \eta^{c}
$$

and $\eta^{c}$ satisfies the conjugated relations

$$
\partial_{\psi} \eta^{c}=-\frac{3 i}{2} \eta^{c}, \quad \tau^{9} \eta^{c}=-\eta^{c}, \quad \tau^{b} J_{a b} \eta^{c}=-i \tau_{a} \eta^{c}, \quad \tau^{b} \bar{\Omega}_{a b} \eta^{c}=0 .
$$

\section{B. IIB spinor decomposition}

We are now in a position to present the fermion decomposition ansatz by expanding the ten-dimensional fermions in terms of $\eta$ and $\eta^{c}$. Although we will ultimately truncate away the massive gravitino multiplet, we find it instructive to start with the complete ansatz. This allows us to identify which fermions belong in which multiplets, and hence will guide the truncation.

Starting with the IIB dilatino, since it has negative chirality, it may be decomposed as ${ }^{1}$

$$
\lambda=e^{-A / 2} \lambda \otimes \eta \otimes\left[\begin{array}{l}
0 \\
1
\end{array}\right]+e^{-A / 2} \lambda^{\prime} \otimes \eta^{c} \otimes\left[\begin{array}{l}
0 \\
1
\end{array}\right] .
$$

The IIB transformation parameter $\epsilon$ and gravitino $\Psi_{A}$ each have positive chirality. Thus we

\footnotetext{
${ }^{1}$ Note that this is a slight abuse of notation, in that $\lambda$ shows up as both ten-dimensional and five-dimensional fields. The correct interpretation will be obvious from the context.
} 
expand the gravitino in ten dimensional flat indices as

$$
\begin{aligned}
& \Psi_{\alpha}=e^{-A / 2} \psi_{\alpha} \otimes \eta \otimes\left[\begin{array}{l}
1 \\
0
\end{array}\right]+e^{-A / 2} \psi_{\alpha}^{\prime} \otimes \eta^{c} \otimes\left[\begin{array}{l}
1 \\
0
\end{array}\right], \\
& \Psi_{a}=e^{-A / 2} \psi \otimes \tau_{a} \eta \otimes\left[\begin{array}{l}
1 \\
0
\end{array}\right]+e^{-A / 2} \psi^{\prime} \otimes \tau_{a} \eta^{c} \otimes\left[\begin{array}{l}
1 \\
0
\end{array}\right], \\
& \Psi_{9}=e^{-A / 2} \psi_{9} \otimes \tau_{9} \eta \otimes\left[\begin{array}{l}
1 \\
0
\end{array}\right]+e^{-A / 2} \psi_{9}^{\prime} \otimes \tau_{9} \eta^{c} \otimes\left[\begin{array}{l}
1 \\
0
\end{array}\right],
\end{aligned}
$$

and the transformation parameter as

$$
\epsilon=e^{A / 2} \varepsilon \otimes \eta \otimes\left[\begin{array}{l}
1 \\
0
\end{array}\right]
$$

Note that in all the above we have included relevant warp factors to account for the breathing and squashing modes.

While we have started with a theory with 32 real supercharges, only a quarter of these are preserved in the $\mathrm{AdS}_{5} \times S E_{5}$ background. By focusing on supersymmetries generated by (30), we are thus restricting our study to five-dimensional supersymmetry parameterized by a single Dirac spinor. This corresponds to an $\mathcal{N}=2$ theory, and provides a motivation for us to remove the massive gravitino from subsequent consideration. (If desired, the full spontaneously broken $\mathcal{N}=4$ symmetry may be obtained by introducing an $\varepsilon \otimes \eta^{c}$ component in (30). However, we will not pursue this here.)

\section{Linearized analysis and the $\mathcal{N}=2$ supermultiplet structure}

Before presenting the fermionic reduction, it is instructive to analyze the linearized equations of motion. Doing so allows us to group the effective five-dimensional fermions into the relevant $\mathcal{N}=2$ supermultiplets as highlighted in [5]. We start by noting that the five-dimensional fermions consist of the two gravitini $\psi_{\alpha}$ and $\psi_{\alpha}^{\prime}$, two dilatini $\lambda$ and $\lambda^{\prime}$ and four additional spin-1/2 fields $\psi, \psi^{\prime}, \psi_{9}$ and $\psi_{9}^{\prime}$ arising from the internal components of the ten-dimensional gravitino.

In the linearized theory, the equations are greatly simplified and the fermions satisfy free massive Dirac and Rarita-Schwinger equations. The $\lambda$ and $\lambda^{\prime}$ equations are naturally diagonal and the gravitino equations are diagonalized by the following modes,

$$
\begin{aligned}
& \hat{\psi}_{\alpha}=\psi_{\alpha}+\frac{i}{3} \gamma_{\alpha}\left(4 \psi+\psi_{9}\right), \quad \psi^{m=11 / 2}=4 \psi+\psi_{9}, \quad \psi^{m=-9 / 2}=\psi-\psi_{9}, \\
& \hat{\psi}_{\alpha}^{\prime}=\psi_{\alpha}^{\prime}+\frac{i}{10}\left(\gamma_{\alpha}+2 \nabla_{\alpha}\right)\left(4 \psi^{\prime}+\psi_{9}^{\prime}\right), \quad \psi^{\prime m=5 / 2}=\psi^{\prime}-\psi_{9}^{\prime} .
\end{aligned}
$$




\begin{tabular}{|c|c|c|c|}
\hline$n$ & Multiplet & State & Field \\
\hline 0 & supergraviton & $\begin{array}{l}D(4,1,1)_{0} \\
D\left(3 \frac{1}{2}, 1, \frac{1}{2}\right)_{-1}+D\left(3 \frac{1}{2}, \frac{1}{2}, 1\right)_{1} \\
D\left(3, \frac{1}{2}, \frac{1}{2}\right)_{0}\end{array}$ & $\begin{array}{l}g_{\mu \nu} \\
\hat{\psi}_{\mu} \\
A_{1}+\frac{1}{6} \mathbb{A}_{1}\end{array}$ \\
\hline 0 & $\mathrm{LH}+\mathrm{RH}$ chiral & $\begin{array}{l}D(3,0,0)_{ \pm 2} \\
D\left(3 \frac{1}{2}, \frac{1}{2}, 0\right)_{1}+D\left(3 \frac{1}{2}, 0, \frac{1}{2}\right)_{-1} \\
D(4,0,0)_{0}+D(4,0,0)_{0}\end{array}$ & $\begin{array}{l}b^{m^{2}=-3} \\
\lambda^{\prime} \\
\tau\end{array}$ \\
\hline 1 & $\mathrm{LH}+\mathrm{RH}$ massive gravitino & $\begin{array}{l}D\left(5 \frac{1}{2}, \frac{1}{2}, 1\right)_{1}+D\left(5 \frac{1}{2}, 1, \frac{1}{2}\right)_{-1} \\
D\left(5, \frac{1}{2}, \frac{1}{2}\right)_{0}+D\left(5, \frac{1}{2}, \frac{1}{2}\right)_{0} \\
D(5,0,1)_{2}+D(5,1,0)_{-2} \\
D(6,0,1)_{0}+D(6,1,0)_{0} \\
D\left(4 \frac{1}{2}, 0, \frac{1}{2}\right)_{1}+D\left(4 \frac{1}{2}, \frac{1}{2}, 0\right)_{-1} \\
D\left(5 \frac{1}{2}, 0, \frac{1}{2}\right)_{-1}+D\left(5 \frac{1}{2}, \frac{1}{2}, 0\right)_{1}\end{array}$ & $\begin{array}{l}\hat{\psi}_{\mu}^{\prime} \\
b_{1}^{i} \\
q_{2} \\
b_{2}^{i} \\
\psi^{\prime m=5 / 2} \\
\lambda\end{array}$ \\
\hline 2 & massive vector & $\begin{array}{l}D\left(7, \frac{1}{2}, \frac{1}{2}\right)_{0} \\
D\left(6 \frac{1}{2}, \frac{1}{2}, 0\right)_{-1}+D\left(6 \frac{1}{2}, 0, \frac{1}{2}\right)_{1} \\
D\left(7 \frac{1}{2}, 0, \frac{1}{2}\right)_{-1}+D\left(7 \frac{1}{2}, \frac{1}{2}, 0\right)_{1} \\
D(6,0,0)_{0} \\
D(7,0,0)_{ \pm 2} \\
D(8,0,0)_{0}\end{array}$ & $\begin{array}{l}\mathbb{A}_{1} \\
\psi^{m=-9 / 2} \\
\psi^{m=11 / 2} \\
\sigma \\
b^{m^{2}=21} \\
\rho\end{array}$ \\
\hline
\end{tabular}

TABLE I: Identification of the bosonic and fermionic states in the Kaluza-Klein spectrum with the linearized modes in the reduction.

In all, the linearized modes satisfy,

$$
\begin{array}{ll}
\gamma^{\mu \alpha \beta} \nabla_{\alpha} \hat{\psi}_{\beta}=\frac{3}{2} \gamma^{\mu \alpha} \hat{\psi}_{\alpha}, & \gamma^{\mu \alpha \beta} \nabla_{\alpha} \hat{\psi}_{\beta}^{\prime}=-\frac{7}{2} \gamma^{\mu \alpha} \hat{\psi}_{\alpha}^{\prime}, \\
\gamma^{\alpha} \nabla_{\alpha} \lambda=\frac{7}{2} \lambda, & \gamma^{\alpha} \nabla_{\alpha} \lambda^{\prime}=-\frac{3}{2} \lambda^{\prime}, \\
\gamma^{\alpha} \nabla_{\alpha} \psi^{m=11 / 2}=\frac{11}{2} \psi^{m=11 / 2}, & \gamma^{\alpha} \nabla_{\alpha} \psi^{m=-9 / 2}=-\frac{9}{2} \psi^{m=-9 / 2}, \\
\gamma^{\alpha} \nabla_{\alpha} \psi^{\prime m=5 / 2}=\frac{5}{2} \psi^{\prime m=5 / 2} . &
\end{array}
$$

Note that the massive gravitino obtains its mass by absorbing the spin- $1 / 2$ combination $4 \psi^{\prime}+\psi_{9}^{\prime}$.

As with the fields in the bosonic truncation, we have arrived at a field content which, in 
the case of the round five-sphere, saturates the lowest harmonic in each of the respective Kaluza-Klein towers as determined in [17, 18]. Noting that, in five dimensions, the relation between the conformal weight $\Delta$ and mass $m$ of the fermions is $|m|=\Delta-2$, we can map the fermion fields into $\mathcal{N}=2 \mathrm{AdS}$ multiplets. First, it is straightforward to see that $\hat{\psi}_{\mu}$ has $m=3 / 2$, corresponding to a massless spin-3/2 field in $\operatorname{AdS}_{5}$. Hence it should be identified with the massless gravitino sitting in the supergraviton multiplet. Also at the zeroth Kaluza-Klein level, the $\mathrm{LH}+\mathrm{RH}$ chiral multiplet contains an $m=3 / 2$ fermion which may be identified as $\lambda^{\prime}$. At level $n=1$, the massive gravitino multiplet has three fermions; one spin-3/2 particle with $m=-7 / 2$ corresponding to the massive gravitino $\hat{\psi}_{\mu}^{\prime}$ and two spin-1/2 particles with $m=5 / 2$ corresponding to $\psi^{\prime m=5 / 2}$ and $m=7 / 2$ corresponding to $\lambda$. Finally, at the $n=2$ Kaluza-Klein level, the massive vector multiplet contains two spin-1/2 particles, $\psi^{m=-9 / 2}$ and $\psi^{m=11 / 2}$. These identifications will be further justified by examining the supersymmetry transformations. The complete field content of the supermultiplets is shown in Table I, where the bosonic fields are fully defined in [5].

\section{THE FIVE-DIMENSIONAL THEORY AND $\mathcal{N}=2$ SUPERGRAVITY}

The linearized analysis above demonstrates that the fields $\psi_{\alpha}^{\prime}, \psi^{\prime}, \psi_{9}^{\prime}$ and $\lambda$ belong to the massive gravitino multiplet. We thus proceed with the $\mathcal{N}=2$ truncation by setting these to zero

$$
\psi_{\alpha}^{\prime}=0, \quad \psi^{\prime}=0, \quad \psi_{9}^{\prime}=0, \quad \lambda=0
$$

It is straightforward to show this this is a consistent truncation, provided the bosonic fields in the massive graviton multiplet are set to zero ${ }^{2}$. Moreover, other than just simplifying the resulting equations, this truncation is natural when explicitly discussing $\mathcal{N}=2$ supersymmetry as the massive gravitino should really be thought of as descending from a spontaneously broken $\mathcal{N}=4$ theory.

\footnotetext{
2 The consistency of this truncation in the bosonic sector has been previously shown in [4 $[4]$.
} 


\section{A. Supersymmetry Variations}

We start with the reduction of the IIB supersymmetry variations given in (16). Inserting the fermion ansätze (28), (29) and (30) into the IIB variations, we arrive at the following five-dimensional variations ${ }^{3}$

$$
\begin{aligned}
\delta \hat{\psi}_{\alpha} \equiv & \mathcal{D}_{\alpha} \varepsilon=\left[D_{\alpha}+\frac{i}{24} e^{C-A}\left(\gamma_{\alpha}{ }^{\nu \rho}-4 \delta_{\alpha}{ }^{\nu} \gamma^{\rho}\right)\left(F_{\nu \rho}-2 e^{-2 B-2 C} p_{\nu \rho}\right)\right. \\
& \left.+\frac{1}{12} \gamma_{\alpha}\left(4 e^{A-2 B+C}+6 e^{A-C}-\left(4+\phi_{0}\right) e^{A-4 B-C}\right)\right] \varepsilon \\
& -e^{-2 B}\left(v_{i} f_{\alpha}^{i}-\frac{i}{3} e^{A-C} v_{i} f_{0}^{i} \gamma_{\alpha}\right) \varepsilon^{c}, \\
\delta \psi^{m=11 / 2}= & {\left[-\frac{i}{2} \gamma^{\mu} \partial_{\mu}(4 B+C)-\frac{3}{8} e^{-4 B} \gamma^{\mu} \mathbb{A}_{\mu}+\frac{1}{8} e^{C-A} \gamma^{\mu \nu}\left(F_{\mu \nu}+e^{-2 B-2 C} p_{\mu \nu}\right)-i e^{A-2 B+C}\right.} \\
& \left.-\frac{3 i}{2} e^{A-C}+\frac{5 i}{8}\left(4+\phi_{0}\right) e^{A-4 B-C}\right] \varepsilon+e^{-2 B}\left(\frac{3 i}{4} \gamma^{\mu} v_{i} f_{\mu}^{i}+\frac{7}{4} e^{A-C} v_{i} f_{0}^{i}\right) \varepsilon^{c}, \\
\delta \psi^{m=-9 / 2}= & {\left[-\frac{i}{2} \gamma^{\mu} \partial_{\mu}(B-C)-\frac{1}{4} e^{-4 B} \gamma^{\mu} \mathbb{A}_{\mu}-\frac{1}{8} e^{C-A} \gamma^{\mu \nu}\left(F_{\mu \nu}+e^{-2 B-2 C} p_{\mu \nu}\right)\right.} \\
& \left.-\frac{3 i}{2} e^{A-2 B+C}+\frac{3 i}{2} e^{A-C}\right] \varepsilon+e^{-2 B}\left(\frac{i}{2} \gamma^{\mu} v_{i} f_{\mu}^{i}-\frac{1}{2} e^{A-C} v_{i} f_{0}^{i}\right) \varepsilon^{c}, \\
\delta \lambda^{\prime}= & -\frac{1}{2 \tau_{2}} \gamma^{\mu} \partial_{\mu} \tau \varepsilon^{c}-i e^{-2 B}\left(\gamma^{\mu} v_{i} \bar{f}_{\mu}^{i}-i e^{A-C} v_{i} \bar{f}_{0}^{i}\right) \varepsilon .
\end{aligned}
$$

The gauge covariant derivative $D_{\alpha}$ acting on $\varepsilon$ is given by $D_{\alpha} \equiv \nabla_{\alpha}-\frac{3 i}{2}\left(A_{\alpha}+\frac{1}{6} e^{-4 B} \mathbb{A}_{\alpha}\right)+$ $\frac{i}{4 \tau_{2}} \partial_{\alpha} \tau_{1}$, where the latter term descends from the traditional charge with respect to the $\mathrm{U}(1)$ compensator field, $Q_{M}$, in the ten dimensional IIB theory [15]. Furthermore, we have defined the five-dimensional supercovariant derivative $\mathcal{D}_{\alpha}$ through the gravitino variation in (34).

There are several facts worth noting about these expressions. Firstly, we see that these variations fit nicely into the multiplet structure as presented in Table II In particular, the dilatino variation is built out of $\tau$ and $\bar{v}_{i} f^{i}$, both of which belong to the $\mathrm{LH}+\mathrm{RH}$ chiral multiplet, since the latter corresponds to $b^{m^{2}=-3}$ according to (11). On the other hand, $\delta \psi^{m=11 / 2}$ and $\delta \psi^{m=-9 / 2}$ contain only terms involving fields from the graviton and massive vector multiplets. [Note that the combination $F_{2}+e^{-2 B-2 C} p_{2}$ appearing in (36) and (37) essentially selects the field strength of the massive vector $\mathbb{A}_{1}$, as can be seen from the definition of $p_{2}$ given in (14)]. These observations give further justification for the multiplet structure presented in section $\coprod \mathrm{C}$.

Furthermore, since the breathing mode is $\rho \sim 4 B+C$, and the squashing mode is $\sigma \sim B-C$, we can identify $\psi^{m=11 / 2}$ with the fermionic partner of the breathing mode and

\footnotetext{
3 Note that with the Dirac matrix conventions described in the appendix we have $\epsilon^{c}=i \varepsilon^{c} \otimes \eta^{c} \otimes\left[\begin{array}{l}1 \\ 0\end{array}\right]$.
} 
$\psi^{m=-9 / 2}$ as the fermionic partner of the squashing mode as first demonstrated in [2]. Finally, from the gauge covariant derivative, it is evident that the combination $A_{\mu}+\frac{1}{6} e^{-4 B} \mathbb{A}_{\mu}$ may be identified with the graviphoton, which is consistent with the linearized analysis in [5]. (The combination $F_{2}-2 e^{-2 B-2 C} p_{2}$ appearing in the gravitino variation is similarly the effective graviphoton field strength.)

The gravitino variation (34) is particularly interesting, as we may attempt to read off an $\mathcal{N}=2$ superpotential from the term proportional to $\gamma_{\alpha} \varepsilon$

$$
W=2 e^{A-2 B+C}+3 e^{A-C}-\frac{1}{2}\left(4+\phi_{0}\right) e^{A-4 B-C} .
$$

Recalling the relations $3 A+4 B+C=0$ and $\phi_{0}=-\frac{2 i}{3} \epsilon_{i j}\left(f_{0}^{i} \bar{f}_{0}^{j}-\bar{f}_{0}^{i} f_{0}^{j}\right)$, we see that the scalar potential can be written as

$$
V=2\left(\mathcal{G}^{-1}\right)^{i j} \partial_{i} W \partial_{j} W-\frac{4}{3} W^{2},
$$

where $\left(\mathcal{G}^{-1}\right)^{i j}$ is the inverse scalar metric which can be read off from the scalar kinetic terms in the Lagrangian and $\{i, j\}$ run over all scalars in the theory.

To verify (39), we made use of the fact that the scalar metric given in [5] is composed of three independent components, pertaining to the independent sets of scalars $\{B, C\},\left\{b_{0}^{1}, b_{0}^{2}\right\}$ and $\tau$, with explicit components

$$
\left(\mathcal{G}_{\{B, C\}}^{-1}\right)^{i j}=\frac{1}{16}\left(\begin{array}{cc}
1 & -1 \\
-1 & 7
\end{array}\right), \quad\left(\mathcal{G}_{\left\{b_{0}^{1}, b_{0}^{2}\right\}}^{-1}\right)^{i j}=\frac{e^{4 B}}{4 \tau_{2}}\left(\begin{array}{cc}
1 & \tau_{1} \\
\tau_{1} & |\tau|^{2}
\end{array}\right), \quad \mathcal{G}_{\tau}^{-1}=\tau_{2}^{2} .
$$

Inserting these expressions into (39) then exactly reproduces the scalar potential appearing in the bosonic Lagrangian. This is, however, a somewhat surprising relation as the actual gravitino variation (34) contains not only the term proportional to the superpotential written above, but another term involving $v_{i} f_{0}^{i} \varepsilon^{c}$ where $v_{i} f_{0}^{i}$ is proportional to $b_{0}^{m^{2}=21}$, as indicated in (11). Based on general $\mathcal{N}=2$ gauged supergravity arguments, this should conceivably also contribute to the scalar potential, but is not taken into account by (39). 


\section{B. Equations of Motion}

Turning to the equations of motion, the reduction of the dilatino equation is the most straightforward. After a bit of manipulation, we obtain

$$
\begin{aligned}
0= & {\left[\gamma^{\mu} \mathcal{D}_{\mu}+\frac{i}{8} \gamma^{\mu \nu}\left(e^{C-A} F_{\mu \nu}-2 e^{-A-2 B-C} p_{\mu \nu}\right)-\frac{1}{4}\left(4+\phi_{0}\right) e^{A-4 B-C}+e^{A-2 B+C}+\frac{3}{2} e^{A-C}\right] \lambda^{\prime} } \\
& -e^{-2 B} v_{i}\left[\frac{4}{5} \gamma^{\mu} \bar{f}_{\mu}^{i}+\frac{28 i}{15} \bar{f}_{0}^{i}\right] \psi^{m=11 / 2}-e^{-2 B} v_{i}\left[\frac{4}{5} \gamma^{\mu} \bar{f}_{\mu}^{i}-\frac{4 i}{5} \bar{f}_{0}^{i} e^{A-C}\right] \psi^{m=-9 / 2},
\end{aligned}
$$

where the supercovariant derivative acting on the dilatino is defined by

$$
\mathcal{D}_{\mu} \lambda^{\prime} \equiv D_{\mu} \lambda^{\prime}-K\left(\lambda^{\prime}\right) \hat{\psi}_{\mu}=\left[\nabla_{\mu}+\frac{3 i}{4 \tau_{2}} \partial_{\mu} \tau_{1}+\frac{3 i}{2}\left(A_{\mu}+\frac{1}{6} e^{-4 B} \mathbb{A}_{\mu}\right)\right] \lambda^{\prime}-K\left(\lambda^{\prime}\right) \hat{\psi}_{\mu} .
$$

The supercovariantization term $K\left(\lambda^{\prime}\right)$ acting on $\hat{\psi}_{\mu}$ is given by the right hand side of the dilatino variation (37) with $\varepsilon$ replaced by $\hat{\psi}_{\mu}$ (and similarly $\varepsilon^{c}$ replaced by $\hat{\psi}_{\mu}^{c}$ ).

Starting with the IIB gravitino, we arrive at three equations, corresponding to the $\alpha, a$, and 9 components. After a fair bit of manipulations, and the appropriate redefinitions given in the first line of (31), we obtain the $\psi^{m=11 / 2}$ and $\psi^{m=-9 / 2}$ equations

$$
\begin{aligned}
0= & {\left[\gamma^{\mu} \mathcal{D}_{\mu}+\frac{3 i}{5} e^{-4 B} \gamma^{\mu} \mathbb{A}_{\mu}-\frac{i}{120} e^{C-A} \gamma^{\mu \nu} F_{\mu \nu}-\frac{11 i}{60} e^{-A-2 B-C} \gamma^{\mu \nu} p_{\mu \nu}\right.} \\
& \left.+e^{A}\left(-\frac{17}{12}\left(4+\phi_{0}\right) e^{-4 B-C}+\frac{1}{15} e^{-2 B+C}-\frac{1}{10} e^{-C}\right)\right] \psi^{m=11 / 2} \\
& {\left[\frac{3 i}{5} e^{-4 B} \gamma^{\mu} \mathbb{A}_{\mu}+\frac{i}{5} e^{C-A} \gamma^{\mu \nu} F_{\mu \nu}-\frac{i}{10} e^{-A-2 B-C} \gamma^{\mu \nu} p_{\mu \nu}+e^{A}\left(\frac{12}{5} e^{-2 B+C}-\frac{12}{5} e^{-C}\right)\right] \psi^{m=-9 / 2} } \\
& +v_{i} e^{-2 B}\left[\left(-\frac{2}{5} \gamma^{\mu} f_{\mu}^{i}+\frac{34 i}{15} e^{A-C} f_{0}^{i}\right) \psi^{c m=11 / 2}+\left(\frac{3}{5} \gamma^{\mu} f_{\mu}^{i}-\frac{7 i}{5} e^{A-C} f_{0}^{i}\right) \psi^{c m=-9 / 2}\right] \\
& +\bar{v}_{i} e^{-2 B}\left(\frac{3}{4} \gamma^{\mu} f_{\mu}^{i}+\frac{7 i}{4} e^{A-C} f_{0}^{i}\right) \lambda^{\prime}, \\
0= & {\left[\gamma^{\mu} \mathcal{D}_{\mu}+\frac{2 i}{5} e^{-4 B} \gamma^{\mu} \mathbb{A}_{\mu}-\frac{3 i}{40} e^{C-A} \gamma^{\mu \nu} F_{\mu \nu}-\frac{3 i}{20} e^{-A-2 B-C} \gamma^{\mu \nu} p_{\mu \nu}\right.} \\
& \left.+e^{A}\left(\frac{1}{4}\left(4+\phi_{0}\right) e^{-4 B-C}+\frac{13}{5} e^{-2 B+C}+\frac{9}{20} e^{-C}\right)\right] \psi^{m=-9 / 2} \\
& {\left[\frac{2 i}{5} e^{-4 B} \gamma^{\mu} \mathbb{A}_{\mu}+\frac{2 i}{15} e^{C-A} \gamma^{\mu \nu} F_{\mu \nu}-\frac{i}{15} e^{-A-2 B-C} \gamma^{\mu \nu} p_{\mu \nu}+e^{A}\left(\frac{8}{5} e^{-2 B+C}-\frac{8}{5} e^{-C}\right)\right] \psi^{m=11 / 2} } \\
& +v_{i} e^{-2 B}\left[\left(\frac{2}{5} \gamma^{\mu} f_{\mu}^{i}-\frac{14 i}{5} e^{A-C} f_{0}^{i}\right) \psi^{c m=11 / 2}+\left(-\frac{3}{5} \gamma^{\mu} f_{\mu}^{i}-\frac{3 i}{5} e^{A-C} f_{0}^{i}\right) \psi^{c m=-9 / 2}\right] \\
& +\bar{v}_{i} e^{-2 B}\left(\frac{1}{2} \gamma^{\mu} f_{\mu}^{i}-\frac{i}{2} e^{A-C} f_{0}^{i}\right) \lambda^{\prime} .
\end{aligned}
$$

As in the dilatino case, we have defined the supercovariant derivatives

$$
\begin{aligned}
\mathcal{D}_{\mu} \psi^{m=11 / 2} & =\left[\nabla_{\mu}+\frac{i}{4 \tau_{2}} \partial_{\mu} \tau_{1}-\frac{3 i}{2}\left(A_{\mu}+\frac{1}{6} e^{-4 B} \mathbb{A}_{\mu}\right)\right] \psi^{m=11 / 2}-K\left(\psi^{m=11 / 2}\right) \hat{\psi}_{\mu}, \\
\mathcal{D}_{\mu} \psi^{m=-9 / 2} & =\left[\nabla_{\mu}+\frac{i}{4 \tau_{2}} \partial_{\mu} \tau_{1}-\frac{3 i}{2}\left(A_{\mu}+\frac{1}{6} e^{-4 B} \mathbb{A}_{\mu}\right)\right] \psi^{m=-9 / 2}-K\left(\psi^{m=-9 / 2}\right) \hat{\psi}_{\mu},
\end{aligned}
$$


with $K\left(\psi^{m=11 / 2}\right)$ and $K\left(\psi^{m=-9 / 2}\right)$ similarly obtained from the variations (36) and (37), respectively.

Finally, the gravitino equation takes the form

$$
0=\gamma^{\mu \nu \rho} \mathcal{D}_{\nu} \hat{\psi}_{\rho}-\frac{8}{15} \tilde{K}\left(\psi^{m=11 / 2}\right) \gamma^{\mu} \psi^{m=11 / 2}-\frac{4}{5} \tilde{K}\left(\psi^{m=-9 / 2}\right) \gamma^{\mu} \psi^{m=-9 / 2}-\frac{1}{2} \tilde{K}\left(\lambda^{\prime}\right) \gamma^{\mu} \lambda^{\prime}
$$

where the supercovariant derivative acting on the gravitino is given by the right hand side

of the gravitino variation (34), and where the $\tilde{K}$ terms are essentially the Dirac conjugates of $K$. The above equations have the appropriate structure to be obtained from an effective $\mathcal{N}=2$ Lagrangian of the form ${ }^{4}$

$$
\begin{aligned}
e^{-1} \mathcal{L}= & \overline{\hat{\psi}}_{\mu} \gamma^{\mu \nu \rho} \mathcal{D}_{\nu} \hat{\psi}_{\rho}+\frac{8}{15} \bar{\psi}^{m=11 / 2} \gamma^{\mu} D_{\mu} \psi^{m=11 / 2}+\frac{4}{5} \bar{\psi}^{m=-9 / 2} \gamma^{\mu} D_{\mu} \psi^{m=-9 / 2}+\frac{1}{2} \bar{\lambda}^{\prime} \gamma^{\mu} D_{\mu} \lambda^{\prime} \\
& +\left[\overline{\hat{\psi}}_{\mu}\left(-\frac{8}{15} \tilde{K}\left(\psi^{m=11 / 2}\right) \gamma^{\mu} \psi^{m=11 / 2}-\frac{4}{5} \tilde{K}\left(\psi^{m=-9 / 2}\right) \gamma^{\mu} \psi^{m=-9 / 2}-\frac{1}{2} \tilde{K}\left(\lambda^{\prime}\right) \gamma^{\mu} \lambda^{\prime}\right)+\text { h.c. }\right] \\
& +\cdots
\end{aligned}
$$

The full fermionic Lagrangian (to quadratic order in the fermions) is given in Appendix B.

Although we have worked only to quadratic order in the fermions, it is clear from the nature of the invariant spinors $\eta$ and $\eta^{c}$ that higher spinor harmonics would not be excited by this subset of states. Thus, if desired, the consistent truncation may be extended to the four-fermi terms as well. However, we expect this to be quite tedious and not particularly worth pursuing.

\section{A SUPERSYMMETRIC HOLOGRAPHIC SUPERCONDUCTOR}

In this final section we demonstrate the consistency of a particularly interesting truncation to the lowest Kaluza-Klein level, namely the supersymmetric completion of the bosonic truncation first demonstrated in [12]. As we demonstrate, this is a fully consistent truncation, so long as we keep all fields in the graviton and LH+RH chiral multiplets. However, it is a nontrivial truncation, in that it is not consistent to naively set the other fields in the above reduction to zero. Instead, the "backreaction" on the truncated fields must be taken

\footnotetext{
${ }^{4}$ Note that some care must be taken when considering the conjugate spinor terms. Nevertheless, the various conjugate terms do assemble themselves properly into a consistent effective fermionic Lagrangian. This is one place where a more conventional symplectic-Majorana approach would allow the manipulations to be more transparent.
} 
into account, effectively setting these modes equal to something depending on the dynamical fields. Due to this backreaction on the non dynamical fields, the resulting Lagrangian is nonlinear and so describes a non-trivial coupling of $\mathcal{N}=2$ supergravity with a single hypermultiplet.

In the bosonic sector the truncation amounts to keeping only $\left\{\tau, b^{m^{2}=-3}\right\}$ and the graviton and graviphoton dynamical. In what follows, we will denote $b^{m^{2}=-3}$ simply as $b$ so that $\left(b_{0}^{1}, b_{0}^{2}\right)=(b, \tau b)$. This requires the following constraints on the other terms in the reduction $[4,5]$

$$
b^{m^{2}=21}=0, \quad e^{4 B}=e^{-4 C}=1-4 \tau_{2}|b|^{2}, \quad \mathbb{A}_{1}=-4 i \tau_{2}(b D \bar{b}-\bar{b} D b)+4|b|^{2} d \tau_{1},
$$

and

$$
\phi_{0}=-24 \tau_{2}|b|^{2}, \quad p_{2}=-d A_{1} .
$$

For the fermions, by analyzing the supersymmetry transformations of the spin- $\frac{1}{2}$ fields in this truncation, it is evident that if we set

$$
\psi=-\psi_{9}=-\frac{i}{2} b \tau_{2}^{1 / 2} e^{-2 B} \lambda^{\prime}
$$

the resulting system will be consistent with the supersymmetry transformations. It turns out that under this identification the fermion equations of motion also degenerate into a single expression, resulting in a theory containing only $\lambda^{\prime}$ and $\hat{\psi}_{\mu}$ in the fermionic sector.

Moving directly to the Lagrangian, we write this as a sum of bosonic and fermionic contributions $\mathcal{L}=\mathcal{L}_{b}+\mathcal{L}_{f}$, where

$$
\begin{aligned}
\mathcal{L}_{b}= & R * 1+\frac{6(2-3 \chi)}{(1-\chi)^{2}} * 1-\frac{d \chi \wedge * d \chi}{2(1-\chi)^{2}}-\frac{(1+\chi) d \tau \wedge * d \bar{\tau}}{2(1-\chi) \tau_{2}^{2}}-\frac{3}{2} F_{2} \wedge * F_{2}-\frac{\mathbb{A}_{1} \wedge * \mathbb{A}_{1}}{2(1-\chi)^{2}} \\
& -\frac{8 \tau_{2} D b \wedge * D \bar{b}}{1-\chi}-\frac{2 i}{1-\chi}(\bar{b} D b \wedge * d \bar{\tau}-b D \bar{b} \wedge * d \tau)-A_{1} \wedge F_{2} \wedge F_{2}
\end{aligned}
$$

and

$$
\begin{aligned}
e^{-1} \mathcal{L}_{f}= & \overline{\hat{\psi}}_{\alpha} \gamma^{\alpha \beta \sigma} D_{\beta} \hat{\psi}_{\sigma}+\frac{3 i}{8} \overline{\hat{\psi}}_{\alpha}\left(\gamma^{\alpha \beta \rho \sigma}+2 g^{\alpha \beta} g^{\rho \sigma}\right) F_{\beta \rho} \hat{\psi}_{\sigma}+\frac{1}{2} \overline{\tilde{\lambda}} \gamma^{\alpha} D_{\alpha} \tilde{\lambda}+\frac{3 i}{16} \overline{\tilde{\lambda}} \gamma^{\mu \nu} F_{\mu \nu} \tilde{\lambda} \\
& +\frac{1}{2} e^{-4 B}\left(3 \tau_{2}\left(b \overline{D_{\mu}} b-\bar{b} D_{\mu} b\right) \overline{\tilde{\lambda}} \gamma^{\mu} \tilde{\lambda}+\frac{3}{2}\left(1+8 \tau_{2}|b|^{2}\right) \overline{\tilde{\lambda}} \tilde{\lambda}\right) \\
& +e^{-4 B}\left(-\frac{3}{2} \overline{\hat{\psi}}_{\alpha} \gamma^{\alpha \sigma} \hat{\psi}_{\sigma}+\tau_{2}\left(\bar{b} D_{\beta} b-b \bar{D}_{\beta} b\right) \overline{\hat{\psi}}_{\alpha} \gamma^{\alpha \beta \sigma} \hat{\psi}_{\sigma}\right) \\
& +\tau_{2}^{1 / 2} e^{-4 B}\left(D_{\mu} b \overline{\hat{\psi}}_{\alpha} \gamma^{\mu} \gamma^{\alpha} \tilde{\lambda}+3 b \overline{\hat{\psi}}_{\alpha} \gamma^{\alpha} \tilde{\lambda}+\text { h.c. }\right) \\
& +\frac{e^{-2 B}}{\tau_{2}^{1 / 2}}\left(-b \overline{\hat{\psi}_{\alpha}} \gamma^{\alpha \beta \sigma} \partial_{\beta} \tau \hat{\psi}_{\sigma}^{c}+\tau_{2}^{1 / 2} \overline{\hat{\psi}}_{\alpha} \gamma^{\mu} \partial_{\mu} \tau \gamma^{\alpha} \tilde{\lambda}^{c}+\text { h.c. }\right)
\end{aligned}
$$


where we have defined $\tilde{\lambda} \equiv e^{-2 B} \lambda^{\prime}, \chi=\tau_{2}|b|^{2}$ and we have redefined the gauge covariant derivative acting on $b$ as $D_{\mu} b=\left(\partial_{\mu}-3 i A_{\mu}-\frac{i}{2 \tau_{2}} \partial_{\mu} \tau_{1}\right) b$, and similarly for $\tilde{\lambda}$ and $\hat{\psi}_{\alpha}$.

This truncation is of interest for many of the condensed matter applications of the AdS/CFT correspondence involving the coupling of a charged scalar and fermion. In particular the original motivation for the bosonic truncation was in describing a superconducting phase transition using holographic methods within a controlled system, i.e, one which is derived directly from a UV complete theory. This truncation hence completes the story by demonstrating the embedding into a fully supersymmetric theory. It would be interesting to consider the dynamics of this theory, and whether there is a supersymmetric superconducting phase transition. Note however that this analysis would be complicated by the presence of the gravitino. After all, it is not consistent to simply set the gravitino field defined here to be zero. Since the gravitino couples to the supercurrent, this suggests that the holographic superconductor model of [12] in fact has an underlying (although spontaneously broken) supersymmetry.

While the truncation first presented in [12] did not include the axi-dilaton, as in the bosonic case, it is consistent to fix $\tau$ as well. This simplifies the Lagrangian to be

$$
\begin{aligned}
e^{-1} \mathcal{L}= & R-\frac{3}{4} F_{\mu \nu} F^{\mu \nu}-e^{-1} A_{1} \wedge F_{2} \wedge F_{2} \\
& +12 \frac{\left(1-6 f^{2}\right)}{\left(1-4 f^{2}\right)^{2}}-8 \frac{\partial_{\mu} f \partial^{\mu} f}{\left(1-4 f^{2}\right)^{2}}-8 f^{2} \frac{\left(\partial_{\mu} \theta-3 A_{\mu}\right)\left(\partial^{\mu} \theta-3 A^{\mu}\right)}{\left(1-4 f^{2}\right)^{2}} \\
& +\overline{\hat{\psi}}_{\alpha} \gamma^{\alpha \beta \sigma} D_{\beta} \hat{\psi}_{\sigma}+\frac{1}{2} \overline{\tilde{\lambda}} \gamma^{\alpha} D_{\alpha} \tilde{\lambda}+\frac{3 i}{8} \overline{\hat{\psi}}_{\alpha}\left(\gamma^{\alpha \beta \rho \sigma}+2 g^{\alpha \beta} g^{\rho \sigma}\right) F_{\beta \rho} \hat{\psi}_{\sigma}+\frac{3 i}{16} \overline{\tilde{\lambda}} \gamma^{\mu \nu} F_{\mu \nu} \tilde{\lambda} \\
& +\frac{1}{1-4 f^{2}}\left(\frac{3}{4}\left(1+8 f^{2}\right) \overline{\tilde{\lambda}} \tilde{\lambda}-\frac{3}{2} \overline{\hat{\psi}}_{\alpha} \gamma^{\alpha \sigma} \hat{\psi}_{\sigma}-i f^{2}\left(\partial_{\mu} \theta-3 A_{\mu}\right)\left(3 \overline{\tilde{\lambda}} \gamma^{\mu} \tilde{\lambda}+2 \overline{\hat{\psi}}_{\alpha} \gamma^{\alpha \beta \sigma} \hat{\psi}_{\sigma}\right)\right) \\
& +\left(\frac{e^{i \theta}}{1-4 f^{2}}\left(\left(\partial_{\mu} f+i f\left(\partial_{\mu} \theta-3 A_{\mu}\right)\right) \overline{\hat{\psi}}_{\alpha} \gamma^{\mu} \gamma^{\alpha} \tilde{\lambda}+3 f \overline{\hat{\psi}}_{\alpha} \gamma^{\alpha} \tilde{\lambda}\right)+h . c .\right)
\end{aligned}
$$

where we have defined $b=\sqrt{g_{s}} f e^{i \theta}$ and $\tau=i g_{s}^{-1}$.

Finally, it is worth noting that although this theory involves a charged scalar coupled to the fermion $\tilde{\lambda}$, it lacks the Majorana coupling $\phi \lambda \lambda$ that has been of recent interest in studies involving gapped fermions in the bosonic condensate [19 21]. While this coupling is allowed by charge conservation, the explicit reduction shows that it is not present. More generally, examination of Table \ demonstrates that the $b^{m^{2}=21}$ scalar in the massive vector multiplet may have such a coupling, and in fact the equations of motion (43) and (44) show that it is exists for both $\psi^{m=11 / 2}$ and $\psi^{m=-9 / 2}$. It would be curious to see if this $b^{m^{2}=21}$ scalar may play a role in novel models of holographic superconductors. 


\section{Acknowledgments}

We wish to thank I. Bah, A. Faraggi, N. Halmagyi and D. Vaman for useful discussions. This work was supported in part by the US Department of Energy under grant DE-FG0295ER40899.

\section{Appendix A: Dirac Matrix Conventions}

We work with a mostly plus metric signature, and take the conventional Clifford algebra $\left\{\Gamma^{A}, \Gamma^{B}\right\}=2 \eta^{A B}$. Note, in particular, that $\Gamma^{0}$ is anti-hermitian, so that $\left(\Gamma^{0}\right)^{\dagger}=-\Gamma^{0}$ and $\left(\Gamma^{i}\right)^{\dagger}=\Gamma^{i}$. The ten-dimensional Chirality matrix is given by

$$
\Gamma^{11} \equiv \frac{1}{10 !} \epsilon_{A_{1} \cdots A_{10}} \Gamma^{A_{1}} \cdots \Gamma^{A_{10}}=\Gamma^{0} \cdots \Gamma^{9}
$$

and squares to the identity.

Corresponding to the metric reduction (44), we decompose the ten-dimensional Dirac matrices according to

$$
\begin{aligned}
& \Gamma^{\alpha} \equiv \gamma^{\alpha} \otimes 1_{4} \otimes \sigma_{1}, \\
& \Gamma^{a} \equiv 1_{4} \otimes \tau^{a} \otimes \sigma_{2}, \\
& \Gamma^{9} \equiv 1_{4} \otimes \tau^{9} \otimes \sigma_{2},
\end{aligned}
$$

where $\gamma^{\alpha}$ are Dirac matrices in the five-dimensional spacetime with $\gamma^{4} \equiv i \gamma^{0} \gamma^{1} \gamma^{2} \gamma^{3}$ and $\tau^{a}$ are Dirac matrices in the five-dimensional internal space with $\tau^{9} \equiv \tau^{5} \tau^{6} \tau^{7} \tau^{8}$. The Chirality matrix $\Gamma^{11}$ is then given by

$$
\Gamma^{11}=\Gamma^{0} \cdots \Gamma^{9}=1_{4} \otimes 1_{4} \otimes \sigma_{3} .
$$

We furthermore take the following conventions for the $A, C$ and $D$ intertwiners which map between different representations of the Dirac matrices

$$
A_{10} \Gamma_{M} A_{10}^{-1}=\Gamma_{M}^{\dagger}, \quad C_{10}^{-1} \Gamma_{M} C_{10}=-\Gamma_{M}^{T}, \quad D_{10}^{-1} \Gamma_{M} D_{10}=-\Gamma_{M}^{*} .
$$

Here $C_{10}$ denotes the charge conjugation matrix. These may be decomposed as

$$
A_{10}=A_{4,1} \otimes A_{5} \otimes \sigma_{1}, \quad C_{10}=C_{4,1} \otimes C_{5} \otimes \sigma_{2}, \quad D_{10}=i D_{4,1} \otimes D_{5} \otimes \sigma_{3},
$$


where the five-dimensional intertwiners are defined as

$$
\begin{array}{rll}
A_{4,1} \gamma_{\mu} A_{4,1}^{-1}=-\gamma_{\mu}^{\dagger}, & C_{4,1}^{-1} \gamma_{\mu} C_{4,1}=\gamma_{\mu}^{T}, & D_{4,1}^{-1} \gamma_{\mu} D_{4,1}=-\gamma_{\mu}^{*} \\
A_{5} \tau_{a} A_{5}^{-1}=\tau_{a}^{\dagger}, & C_{5}^{-1} \tau_{a} C_{5}=\tau_{a}^{T}, & D_{5}^{-1} \tau_{a} D_{5}=\tau_{a}^{*} .
\end{array}
$$

It turns out the following is a consistent decomposition:

$$
A_{10}=\Gamma_{0}=\gamma_{0} \otimes 1 \otimes \sigma_{1}, \quad C_{10}=C_{4,1} \otimes C_{5} \otimes \sigma_{2}, \quad D_{10}=i \gamma_{0} C_{4,1} \otimes C_{5} \otimes \sigma_{3} .
$$

The five dimensional charge conjugation matrices on both spacetime and the internal manifold satisfy

$$
C_{5}=-C_{5}^{T}=C_{5}^{*}=-C_{5}^{-1} .
$$

Finally, we define the charge conjugate of a spinor in any dimension to be $\psi^{c}=C A^{T} \psi^{*}$, which is equivalent to $\psi^{c}=-\Gamma_{0} C_{10} \psi^{*}$. Therefore, letting $\chi$ and $\eta$ be spinors on $M$ and $S E_{5}$, respectively, the charge conjugates are given by $\chi^{c}=-\gamma_{0} C_{4,1} \chi^{*}$ and $\eta^{c}=C_{5} \eta^{*}$.

\section{Appendix B: The Reduced Lagrangian}

The bosonic Lagrangian with the massive gravitino multiplet removed was presented in [5], and takes the form

$$
\begin{aligned}
\mathcal{L}_{b}= & R * 1+\left(24 e^{2 A-2 B}-4 e^{5 A+3 C}-\frac{1}{2} e^{8 A}\left(4+\phi_{0}\right)^{2}\right) * 1-\frac{28}{3} d B \wedge * d B-\frac{8}{3} d B \wedge * d C \\
& -\frac{4}{3} d C \wedge * d C-\frac{1}{2 \tau_{2}^{2}} d \tau \wedge * d \bar{\tau}-\frac{1}{2} e^{2 C-2 A} F_{2} \wedge * F_{2}-e^{A-C}\left(F_{2}+\frac{1}{4} \mathbb{F}_{2}^{\prime}\right) \wedge *\left(F_{2}+\frac{1}{4} \mathbb{F}_{2}^{\prime}\right) \\
& -\frac{1}{2} e^{-8 B}\left[\mathbb{A}_{1}^{\prime}-\frac{2 i}{3} \epsilon_{i j}\left(f_{0}^{i} \bar{f}_{1}^{j}-\bar{f}_{0}^{i} f_{1}^{j}\right)\right] \wedge *\left[\mathbb{A}_{1}^{\prime}-\frac{2 i}{3} \epsilon_{i j}\left(f_{0}^{i} \bar{f}_{1}^{j}-\bar{f}_{0}^{i} f_{1}^{j}\right)\right] \\
& -2 \mathcal{M}_{i j}\left[e^{5 A-C}\left(f_{0}^{i} \bar{f}_{0}^{j}+\bar{f}_{0}^{i} f_{0}^{j}\right) * 1+e^{-4 B}\left(f_{1}^{i} \wedge * \bar{f}_{1}^{j}+\bar{f}_{1}^{i} \wedge * f_{1}^{j}\right)\right] \\
& -A_{1} \wedge\left(F_{2}+\frac{1}{4} \mathbb{F}_{2}^{\prime}\right) \wedge\left(F_{2}+\frac{1}{4} \mathbb{F}_{2}^{\prime}\right),
\end{aligned}
$$

where $\mathbb{A}_{1}^{\prime}=\mathbb{A}_{1}+\frac{2 i}{3} \epsilon_{i j}\left(f_{0}^{i} \bar{f}_{1}^{j}-\bar{f}_{0}^{i} f_{1}^{j}\right)$, and where $\mathbb{F}_{2}^{\prime}=d \mathbb{A}_{1}^{\prime}$.

The corresponding fermionic Lagrangian may be obtained from the equations of motion 
presented in Section IVB. At quadratic order in the fermions, we have

$$
\begin{aligned}
& e^{-1} \mathcal{L}_{f}= \overline{\hat{\psi}}_{\mu} \gamma^{\mu \nu \rho} \mathcal{D}_{\nu} \hat{\psi}_{\rho} \\
&+\left[-\frac{8}{15} \bar{\psi}^{m=11 / 2} \gamma^{\mu} K\left(\psi^{m=11 / 2}\right) \hat{\psi}_{\mu}-\frac{4}{5} \bar{\psi}^{m=-9 / 2} \gamma^{\mu} K\left(\psi^{m=-9 / 2}\right) \hat{\psi}_{\mu}\right. \\
&\left.\quad-\frac{1}{2} \bar{\lambda}^{\prime} \gamma^{\mu} K\left(\lambda^{\prime}\right) \hat{\psi}_{\mu}+h . c .\right] \\
&+\frac{8}{15} \bar{\psi}^{m=11 / 2}\left[\gamma^{\mu} D_{\mu}+\frac{3 i}{5} e^{-4 B} \gamma^{\mu} \mathbb{A}_{\mu}-\frac{i}{120} e^{C-A} \gamma^{\mu \nu} F_{\mu \nu}-\frac{11 i}{60} e^{-A-2 B-C} \gamma^{\mu \nu} p_{\mu \nu}\right. \\
&\left.+e^{A}\left(-\frac{17}{12}\left(4+\phi_{0}\right) e^{-4 B-C}+\frac{1}{15} e^{-2 B+C}-\frac{1}{10} e^{-C}\right)\right] \psi^{m=11 / 2} \\
&+\frac{4}{5} \psi^{m=-9 / 2}\left[\gamma^{\mu} D_{\mu}+\frac{2 i}{5} e^{-4 B} \gamma^{\mu} \mathbb{A}_{\mu}-\frac{3 i}{40} e^{C-A} \gamma^{\mu \nu} F_{\mu \nu}-\frac{3 i}{20} e^{-A-2 B-C} \gamma^{\mu \nu} p_{\mu \nu}\right. \\
&\left.+\frac{1}{2} \bar{\lambda}^{\prime}\left[\gamma^{\mu} D_{\mu}+\frac{1}{4}\left(4+\phi_{0}\right) e^{-4 B-C}+\frac{13}{5} e^{-2 B+C}+\frac{9}{20} e^{-C}\right)\right] \psi^{m=-9 / 2} \\
& \quad-\frac{1}{4}\left(4+e^{C-A} F_{\mu \nu}-2 e^{-A-2 B-C} p_{\mu \nu}\right) \\
&+\frac{8}{15}\left[\overline { \psi } ^ { m = 1 1 / 2 } \left(\frac{3 i}{5} e^{-4 B} \gamma^{\mu} \mathbb{A}_{\mu}+\frac{i}{5} e^{C-A} \gamma^{\mu \nu} F_{\mu \nu}-\frac{i}{10} e^{-A-2 B-C} \gamma^{\mu \nu} p_{\mu \nu}\right.\right. \\
&\left.\left.+\frac{4}{A}\left(\frac{12}{5} e^{-2 B+C}-\frac{12}{5} e^{-C}\right)\right) \psi^{m=-9 / 2}+h . c .\right] \\
&+\frac{4}{5}\left[\bar{v}_{i} e^{-2 B} \bar{\psi}^{m=-9 / 2}\left(\frac{1}{2} \gamma^{\mu} f_{\mu}^{i}-\frac{i}{2} e^{A-C} f_{0}^{i}\right) \lambda^{\prime}+h . c .\right] \\
&+\frac{8}{5}\left[v_{i} e^{-2 B} \bar{\psi}^{m=11 / 2}\left(-\frac{2}{5} \gamma^{\mu} f_{\mu}^{i}+\frac{34 i}{15} e^{A-C} f_{0}^{i}\right) \psi^{c m=11 / 2}+h . c .\right] \\
&+\frac{8}{15}\left[v_{i} e^{-2 B} \bar{\psi}^{m=11 / 2}\left(\frac{3}{5} \gamma^{\mu} f_{\mu}^{i}-\frac{7 i}{5} e^{A-C} f_{0}^{i}\right) \psi^{c m=-9 / 2}+h . c .\right] \\
&\left.\left.+-\frac{3}{5} \gamma^{\mu} f_{\mu}^{i}-\frac{3 i}{5} e^{A-C} f_{0}^{i}\right) \psi^{c m=-9 / 2}+h . c .\right] \\
&\left.\bar{v}^{-2 B} \bar{\psi}^{m=11 / 2}\left(\frac{3}{4} \gamma^{\mu} f_{\mu}^{i}+\frac{7 i}{4} e^{A-C} f_{0}^{i}\right) \lambda^{\prime}+h . c .\right]
\end{aligned}
$$

and the full Lagrangian up to quadratic order in the fermions is given by

$$
\mathcal{L}=\mathcal{L}_{b}+\mathcal{L}_{f}
$$

[1] M. S. Bremer, M. J. Duff, H. Lu, C. N. Pope and K. S. Stelle, Instanton cosmology and domain walls from M-theory and string theory, Nucl. Phys. B 543, 321 (1999) arXiv:hep-th/9807051.

[2] J. T. Liu and H. Sati, Breathing mode compactifications and supersymmetry of the braneworld, Nucl. Phys. B 605, 116 (2001) arXiv:hep-th/0009184.

[3] J. P. Gauntlett, S. Kim, O. Varela and D. Waldram, Consistent supersymmetric Kaluza-Klein truncations with massive modes, JHEP 0904, 102 (2009) [arXiv:0901.0676 [hep-th]]. 
[4] D. Cassani, G. Dall'Agata and A. F. Faedo, Type IIB supergravity on squashed Sasaki-Einstein manifolds, JHEP 1005, 094 (2010) [arXiv:1003.4283 [hep-th]].

[5] J. T. Liu, P. Szepietowski and Z. Zhao, Consistent massive truncations of IIB supergravity on Sasaki-Einstein manifolds, Phys. Rev. D 81, 124028 (2010) arXiv:1003.5374 [hep-th]].

[6] J. P. Gauntlett and O. Varela, Universal Kaluza-Klein reductions of type IIB to $\mathcal{N}=4$ supergravity in five dimensions, JHEP 1006, 081 (2010) [arXiv:1003.5642 [hep-th]].

[7] K. Skenderis, M. Taylor and D. Tsimpis, A consistent truncation of IIB supergravity on manifolds admitting a Sasaki-Einstein structure, JHEP 1006, 025 (2010) arXiv:1003.5657 [hep-th]].

[8] D. Cassani and A. F. Faedo, A supersymmetric consistent truncation for conifold solutions, arXiv:1008.0883 [hep-th].

[9] I. Bena, G. Giecold, M. Grana, N. Halmagyi and F. Orsi, Supersymmetric Consistent Truncations of IIB on $T^{1,1}$, arXiv:1008.0983 [hep-th].

[10] J. P. Gauntlett, J. Sonner and T. Wiseman, Holographic superconductivity in M-Theory, Phys. Rev. Lett. 103, 151601 (2009) [arXiv:0907.3796 [hep-th]].

[11] J. P. Gauntlett, J. Sonner and T. Wiseman, Quantum Criticality and Holographic Superconductors in M-theory, JHEP 1002, 060 (2010) [arXiv:0912.0512 [hep-th]].

[12] S. S. Gubser, C. P. Herzog, S. S. Pufu and T. Tesileanu, Superconductors from Superstrings, Phys. Rev. Lett. 103, 141601 (2009) [arXiv:0907.3510 [hep-th]].

[13] I. Bah, A. Faraggi, J. I. Jottar, R. G. Leigh and L. A. P. Zayas, Fermions and D $=11$ Supergravity On Squashed Sasaki-Einstein Manifolds, arXiv:1008.1423 [hep-th].

[14] I. Bah, A. Faraggi, J. I. Jottar and R. G. Leigh, Fermions and Type IIB Supergravity On Squashed Sasaki-Einstein Manifolds, arXiv:1009.1615 [hep-th].

[15] J. H. Schwarz, Covariant Field Equations Of Chiral $N=2 D=10$ Supergravity, Nucl. Phys. B 226, 269 (1983).

[16] G. W. Gibbons, S. A. Hartnoll and C. N. Pope, Bohm and Einstein-Sasaki metrics, black holes and cosmological event horizons, Phys. Rev. D 67, 084024 (2003) arXiv:hep-th/0208031.

[17] M. Gunaydin and N. Marcus, The Spectrum Of The $S^{5}$ Compactification Of The Chiral $N=2, D=10$ Supergravity And The Unitary Supermultiplets Of U(2,2/4), Class. Quant. Grav. 2, L11 (1985).

[18] H. J. Kim, L. J. Romans and P. van Nieuwenhuizen, The Mass Spectrum Of Chiral $N=2$ 
$D=10$ Supergravity On $S^{5}$, Phys. Rev. D 32, 389 (1985).

[19] J. W. Chen, Y. J. Kao and W. Y. Wen, Peak-Dip-Hump from Holographic Superconductivity, Phys. Rev. D 82, 026007 (2010) arXiv:0911.2821 [hep-th]].

[20] T. Faulkner, G. T. Horowitz, J. McGreevy, M. M. Roberts and D. Vegh, Photoemission 'experiments' on holographic superconductors, JHEP 1003, 121 (2010) arXiv:0911.3402 [hepth]].

[21] S. S. Gubser, F. D. Rocha and P. Talavera, Normalizable fermion modes in a holographic superconductor, arXiv:0911.3632 [hep-th]. 